\title{
Domain wall properties of FePt: From Bloch to linear walls
}

\author{
D. Hinzke, ${ }^{1, *}$ N. Kazantseva, ${ }^{1}$ U. Nowak, ${ }^{1}$ O. N. Mryasov, ${ }^{2}$ P. Asselin, ${ }^{2}$ and R. W. Chantrell ${ }^{1}$ \\ 'Department of Physics, University of York, York YOIO SDD, United Kingdom \\ ${ }^{2}$ Seagate Research, 1251 Waterfront Place, Pitssurgh, Pennsylvania 15222, USA
}

\begin{abstract}
An investigation of the orientation and temperature dependence of domain wall properties in FePt is presented. We use a microscopic, atomic model for the magnetic interactions within an effective, classical spin Hamiltonian constructed on the basis of spin density functional theory. We find a significant dependence of the domain wall width and the domain wall energy on the orientation of the wall with respect to the crystal lattice. Investigating the temperature dependence, we demonstrate the existence of elliptical as well as linear domain walls in FePt. The calculation and further analysis of the domain wall free energy results in the evaluation of a thermodynamic exchange stiffness and anisotropy constant.
\end{abstract}

PACS number(s): $75.40 . \mathrm{Mg}, 75.75 .+\mathrm{a}, 75.60 . \mathrm{Ch}$

\section{INTRODUCTION}

Few publications exist in which the temperature dependence of domain wall properties in magnetic materials is investigated. ${ }^{1-10}$ The pioneering work in this context is from Bulaevski and Ginzburg ${ }^{1}$ who showed within the framework of the Ginzburg-Landau theory that for a one-dimensional domain wall profile, such as a Bloch wall, the easy-axis and transverse components of the magnetization are two separate order parameters with different critical temperatures. Consequently, the magnitude of the magnetization in the bulk deviates from the magnitude of the magnetization within the domain wall, leading to the concept of elliptical domain walls. Further, for temperatures between the two critical temperatures, the so-called linear walls ${ }^{1}$ exist, within which the transverse component of the magnetization vanishes while the easy-axis component of the magnetization is still finite. The difference between these two critical temperatures is proportional to the squared inverse domain wall width ${ }^{1,2,5}$ and, as a consequence, the effects described above are very small and hard to detect experimentally. ${ }^{8}$ However, there are cases where these effects might be observable, as, e.g., domain walls in nanoconstrictions, ${ }^{5}$ vortex cores, ${ }^{6}$ or in materials with very large anisotropy?

In the current paper, we focus on FePt in the chemically ordered $L 1_{0}$ phase as a material with very high magnetocrystalline anisotropy energy, a property which is of critical importance for the development of future high density magnetic recording systems. As discussed in Ref. 7, domain wall energies and domain wall widths depend on the orientation of the domain wall with respect to the crystal lattice due to the layered structure of FePt, where the exchange interactions are larger within the $\mathrm{Fe}$ planes than between different $\mathrm{Fe}$ planes.

The main goal of the paper is an investigation of the temperature dependence of the domain wall properties. Using an effective classical spin model for FePt, we perform the Langevin dynamics simulations with which we are able to demonstrate the appearance of elliptical and even linear domain walls in FePt. The computational results are supported by mean-field calculations. Our findings contradict the assumption of a constant magnetization magnitude which is usually made in micromagnetic calculations. A calculation and further analysis of the domain wall free energy makes it possible to evaluate the thermodynamic, i.e., temperature dependent exchange stiffness and anisotropy constant of FePt. These quantities might serve in the future as input parameters for a finite temperature micromagnetic theory. ${ }^{11}$

\section{MODELS AND METHODS}

\section{A. Spin model for FePt}

FePt is modeled in the ordered $L l_{0}$ phase using an effective, classical spin Hamiltonian which was constructed earlier ${ }^{12}$ on the basis of first-principles calculations of noncollinear configurations, calculated using constrained local spin density functional theory ${ }^{13}$ and site-resolved magnetocrystalline anisotropy. ${ }^{14}$ It was found that magnetic interaction parameters are strongly affected by the fact that the magnetic moment of the $5 d$ sites is entirely due to the exchange fields provided by the $3 d$ sites. It was shown that this important feature of the electronic interactions can be taken into account within a model of localized Fe magnetic moments with modified effective magnetic interactions. In addition to the commonly considered Heisenberg exchange and single-ion anisotropy, these modified effective magnetic interactions include an isotropic exchange term dependent on the Pt intra-atomic exchange as well as an effective exchange mediated two-ion anisotropy. ${ }^{12}$ Both isotropic and anisotropic extra terms are positive and thus stabilizing ferromagnetic order along the 001 direction. $^{15}$ The validity of the model was underlined by the fact that it successfully describes the critical temperature and the anomalous temperature dependence of the uniaxial anisotropy energy constant $K_{1}$ found in this ordered alloy. ${ }^{12}$ Furthermore, the same model has been used to investigate the thermally activated switching behavior of FePt nanoparticles..$^{16}$

This model is used here to investigate the domain wall properties of FePt. The full Hamiltonian, described in detail in Ref. 12 , is 


$$
\begin{aligned}
\mathcal{H}= & -\sum_{i<j}\left(J_{i j} \mathbf{S}_{i} \cdot \mathbf{S}_{j}+d_{i j}^{(2)} S_{i}^{z} S_{j}^{z}\right)-\sum_{i} d^{(0)}\left(S_{i}^{z}\right)^{2} \\
& -\sum_{i<j} \frac{\mu_{0} \mu_{s}^{2}}{4 \pi} \frac{3\left(\mathbf{S}_{i} \cdot \mathbf{e}_{i j}\right)\left(\mathbf{e}_{i j} \cdot \mathbf{S}_{j}\right)-\mathbf{S}_{i} \cdot \mathbf{S}_{j}}{r_{i j}^{3}}-\sum_{i} \mu_{s} \mathbf{B} \cdot \mathbf{S}_{i},
\end{aligned}
$$

where the $\mathbf{S}_{i}=\boldsymbol{\mu}_{i} / \mu_{s}$ are three-dimensional reduced magnetic moments of unit length. The first sum represents the exchange energy of magnetic moments, and the two-ion anisotropy is not restricted to nearest-neighbor interactions. The exchange interactions $J_{i j}$ (and consequently also $d_{i j}^{(2)}$ ) have to be taken into account up to a distance of five unit cells until they are finally small enough to be neglected. The two-ion anisotropy parameters $d_{i j}^{(2)}$ are the dominant contribution to the uniaxial anisotropy energy in relation to the single-ion term $d^{(0)}$, which is represented in the second sum. Furthermore, these interactions depend on the orientation in the crystal. The third sum represents the dipole-dipole coupling, while the last one is the coupling of the magnetic moments to an external field, where $\mathbf{B}$ is the induction. Fe-Fe exchange interactions within the Fe planes are stronger than those perpendicular to the planes via the Pt sites. As was shown in Ref. 7, this fact will lead to domain wall properties which depend on the orientation of the domain wall relative to the Fe planes.

For an investigation of domain wall properties, we use a cubic computational cell in zero external field, heating up from $0 \mathrm{~K}$ to above the critical temperature. We force a domain wall into the system by using fixed, antiparallel boundary conditions for two opposite planes and open boundary conditions otherwise. Two orientations of the domain wall are considered where the domain walls are either perpendicular $(\perp)$ or parallel (II) to the Fe planes. ${ }^{7}$ We chose different system sizes, varying the cross section of our system, to investigate finite-size effects. In the following, we show only results for the largest systems we simulated with a cross section of $25.6 \times 25.6 \mathrm{~nm}^{2}$ and a length of $12.8 \mathrm{~nm}$. A more detailed finite-size analysis was carried out earlier ${ }^{5}$ in connection with linear walls in nanoconstrictions. The system length was kept constant but much larger than the zerotemperature domain wall width. Note, however, that since in the critical region the width of the linear domain wall diverges, additional finite-size effects can be expected when the domain wall widths exceed the system length $(12.8 \mathrm{~nm})$.

\section{B. Langevin dynamics simulation}

Since we are interested in ground state properties as well as finite temperatures, we use the Langevin dynamics, i.e., simulations of the stochastic Landau-Lifshitz-Gilbert (LLG) equation of motion. This equation has the form

$$
\frac{\left(1+\alpha^{2}\right) \mu_{s}}{\gamma} \dot{\mathbf{S}}_{i}=-\mathbf{S}_{i} \times \mathbf{H}_{i}(t)-\alpha \mathbf{S}_{i} \times\left[\mathbf{S}_{i} \times \mathbf{H}_{i}(t)\right],
$$

with the gyromagnetic ratio $\gamma=1.76 \times 10^{11}(\mathrm{~T} \mathrm{~s})^{-1}$ and a dimensionless Gilbert damping parameter which we set to $\alpha$ $=1$ (high damping limit). Thermal fluctuations are included as an additional noise term $\zeta_{i}(t)$ in the internal fields $\mathbf{H}_{i}(t)$ $=-\frac{\partial H}{S_{i}}+\zeta_{i}(t)$, with $\left\langle\zeta_{i}(t)=0\right\rangle$ and $\left\langle\zeta_{i}^{k}(t) \zeta_{i}\left(t^{\prime}\right)\right\rangle=2 \delta_{i j} \delta_{k l} \delta(t$ $\left.-t^{\prime}\right) \alpha k_{B} T \mu_{s} / \gamma$, where $i, j$ denotes lattice sites and $k, l$ the Cartesian components. This choice guarantees the correct statistical properties in equilibrium. Note that the value of the damping parameter does not influence thermal equilibrium properties, only the dynamics of the system. The large value for $\alpha$ we chose guarantees fast relaxation to thermal equilibrium and will not influence our results since we are only interested in equilibrium properties. All algorithms we use are described in detail in Ref. 17.

\section{Mean-field approximation}

Even though the methods described in the previous section provide a powerful simulation tool, the investigation is far from being simple. When approaching the critical temperature finite-size effects and strong fluctuations of the wall profiles make the interpretation of the results difficult. Therefore, we also use a mean-field (MF) approximation to confirm our results with a simpler approach which shows less finite-size effects and avoids fluctuations.

In the MF approximation, we assume that all magnetic moments within one plane have the same orientation. In this limit, the model is effectively one dimensional. Furthermore, we simplify our model to a simple cubic lattice with nearestneighbor interaction and a single-ion uniaxial anisotropy only. Based on the MF Hamiltonian,

$$
\begin{aligned}
\mathcal{H}_{\mathrm{MF}}= & -\sum_{i}\left(J \mathbf{S}_{i} \cdot\left(\mathbf{m}_{i-1}+4 \mathbf{m}_{i}+\mathbf{m}_{i+1}\right)+D\left(S_{i}^{z}\right)^{2}\right) \\
& +\frac{1}{2} \sum_{i} J \mathbf{m}_{i} \cdot\left(\mathbf{m}_{i-1}+4 \mathbf{m}_{i}+\mathbf{m}_{i+1}\right),
\end{aligned}
$$

we solve the MF self-consistency equations,

$$
\mathbf{m}_{i}=\left\langle\mathbf{S}_{i}\right\rangle=\frac{1}{Z} \operatorname{Tr} \mathbf{S}_{i} e^{-\beta H_{M F}},
$$

with the partition function $Z=\operatorname{Tr} e^{-\beta H_{\mathrm{MF}}}$ numerically. Here, $\mathbf{m}_{i}$ is the thermally averaged magnetization of the $i$ th plane and the trace is an integral over the unit sphere. We solve this equation iteratively by starting with an arbitrary magnetization profile and letting the equation evolve until a stationary state is reached. A more detailed description of the model can be found in Ref. 5 . For our simulation, we consider a chain with a length of 64 planes and two different values for the anisotropy, namely, $0.2 J$ and $0.01442 J$. The last value for $d / J$ resembles FePt parameters, the other, larger value, is just to show bigger effects for a clearer understanding.

\section{RESULTS}

Here, we study the influence of the temperature on the domain wall properties. There are three types of domain walls one can expect in our model, namely, circular, elliptical, and linear walls. They are sketched in Fig. 1. For the system geometry under investigation, the domain walls are, in general, of Bloch type, with the transverse magnetization in the plane of the wall. In the zero-temperature limit, the walls are circular with constant magnitude of the magnetiza- 

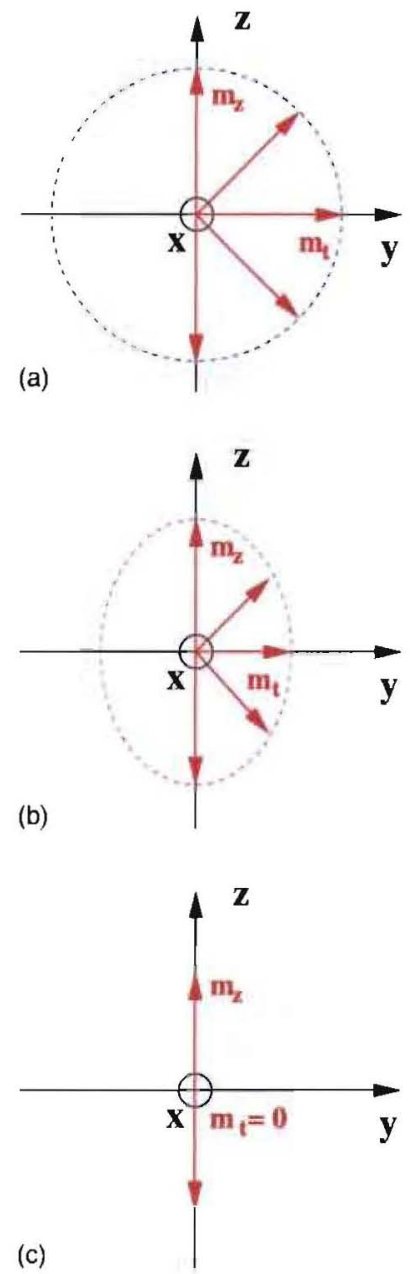

FIG. 1. (Color online) Sketches of the different types of domain wall: (a) circular, (b) elliptical, and (c) linear ones. The $z$ component and the transverse component of the magnetization are shown.

tion. With increasing temperature, the walls become increasingly elliptical with the transverse magnetization being smaller than the bulk value. Near the critical temperature, linear walls are predicted ${ }^{1}$ where the transverse component of the magnetization is zero. These different types of domain wall profiles and their energies are discussed as follows.

\section{A. Domain wall profiles}

The simulations start with a zero-temperature Bloch wall profile in the center of the system which we equilibrate for each temperature. Then, we calculate the easy-axis component of the reduced magnetization $m_{z}$, in addition to the absolute value of the reduced transverse magnetization component (perpendicular to the easy axis) $m_{t}$, averaged over each plane which is parallel to the wall and over a short time interval. Note that the long time average of one transverse magnetization component ( $x$ or $y$ ) might always be zero at higher temperatures since the wall magnetization could rotate in the transverse plane. Hence, calculating its magnitude

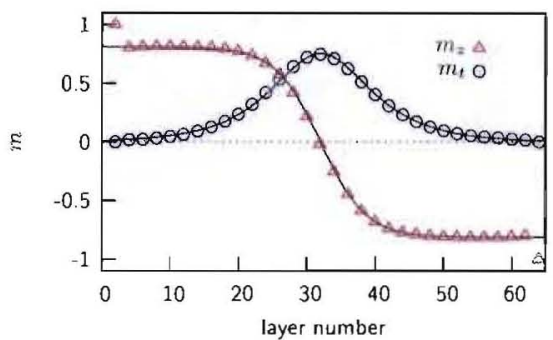

FIG. 2. (Color online) Easy axis and transverse component of the magnetization versus space coordinate for parallel DWs at $311 \mathrm{~K}$. The solid lines are fitted following Eqs. (5) and (6).

is essential. Furthermore, at finite temperatures, the wall position can move diffusively, so that we have to adjust the coordinate system accordingly for the analysis.

Let us start with a general discussion of our domain wall analysis, as shown in Fig. 2. In this figure, the $z$ component (easy axis) of the magnetization determined as explained above is shown in addition to the transverse component. A small deviation from the expected domain wall profiles caused by the fixed boundary conditions can be seen. Hence, in the following, when analyzing the profiles, we focus only on the inner parts, where the domain profiles are not affected by our chosen boundary conditions. Here, the calculated wall profiles were found to be well described by the usual hyperbolic functions (see also Ref. 5),

$$
m_{z}(T)=h_{z}(T) \tanh [x / \delta(T)]
$$

and

$$
m_{l}(T)=h_{t}(T) \cosh ^{-1}[x / \delta(T)],
$$

where $h_{t}$ and $h_{z}$ are the amplitudes of the magnetization and $\delta$ is the domain wall width. Note that both amplitudes as well as wall widths depend on the temperature and the orientation of the wall in the crystal.

We turn now to the analysis of thermal domain wall (DW) properties, calculated first with the full atomistic FePt model. Figure 3 shows the easy-axis $\left(m_{z}\right)$ and transverse $\left(m_{t}\right)$ components of the wall profiles for the perpendicular orientated case for four different temperatures. The domain wall profiles for the parallel case exhibit similar behavior. Figure 4 shows the corresponding results obtained from our MF approximation. Since we show here MF results for a larger anisotropy value, the walls are narrower and temperature dependent effects are stronger. Nevertheless, we obtain the same qualitative behavior with both methods.

In the zero-temperature case, the amplitudes of the domain walls are equal to one $\left(h_{z}=h_{t}=1\right)$. In this case, we find a perfect circular Bloch wall. ${ }^{7}$ Note that for the chosen geometry, Néel walls do not occur and walls are preferentially of Bloch type. However, it can be seen that both amplitudes $\left(h_{z}, h_{l}\right)$ decrease with increasing temperature. Furthermore, the value of the transverse amplitude $h_{t}$ decreases faster than $h_{z}$, so that $h_{\ell}<h_{z}$ for any finite temperature. This is essentially the signature of elliptical walls, and it demonstrates their existence in FePt for finite temperatures (see also 


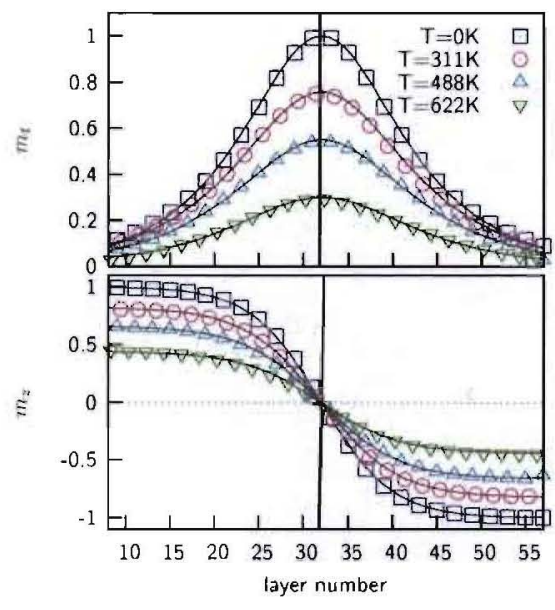

FIG. 3. (Color online) $z$ component (below) and transverse component (above) of the magnetization versus space coordinate for perpendicular DWs at different temperatures. Results are from atomistic simulations for FePt. Solid lines represent fitted cosh and tanh profiles, respectively, to Eqs. (6) and (5).

Fig. 5). With a further increase of temperature, it is found that the transverse component $h_{t}$ vanishes at a certain temperature at which $h_{z}$ remains finite. This indicates the appearance of a so-called linear wall as predicted ${ }^{1}$ near (but still below) the Curie temperature. In our LLG simulation, this behavior is difficult to illustrate due to thermal fluctuations and is not shown in Fig. 3, while the MF approximation calculation shows clearly the appearance of linear walls above $0.95 T_{C}$.

The transitions between the various types of domain walls are better illustrated in terms of the atomistic calculations using the amplitudes $\left(h_{z}, h_{t}\right)$. These are time averaged quantities obtained by fitting to wall profiles and are more statis-

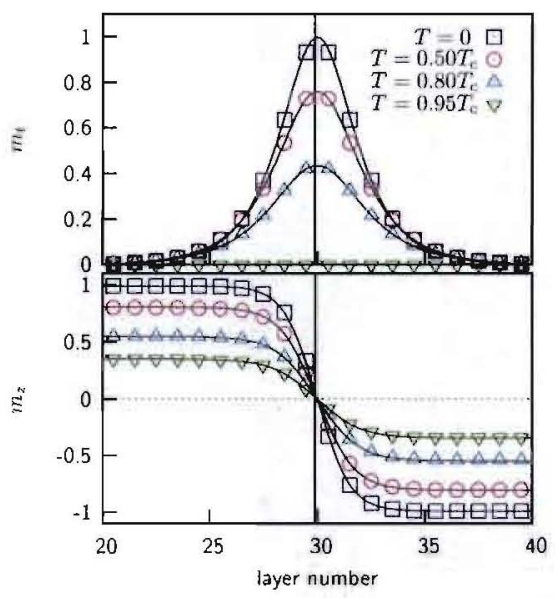

FIG. 4. (Color online) $z$ component (below) and transverse component (above) of the magnetization versus space coordinate. Results are from MF calculations for the larger value of anisotropy. Solid lines represent fitted cosh and tanh profiles, respectively, to Eqs. (6) and (5).
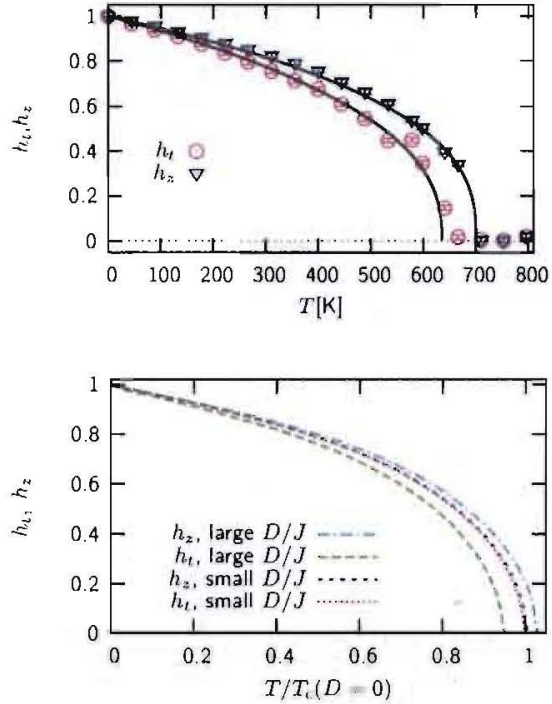

FIG. 5. (Color online) The transverse and easy-axis amplitudes versus temperature. In the upper figure, results are from atomistic simulations for $\mathrm{FePt}$; in the lower figure, results are from MF approximation for two different values of the anisotropy.

tically meaningful than DW profiles at high temperatures, especially given the diffusive DW motion observed in this temperature regime. The results are summarized in Fig. 5, where the fitted amplitudes are presented for the atomistic FePt model and the MF approximation. Once again, Eqs. (6) and (5) are used for fitting the amplitude (Fig. 5) and the domain wall width (Fig. 6). The temperature dependence of the amplitude illustrates the transitions between the different types of domain wall discussed previously. At zero temperature, both amplitudes are identical, as expected. For any fi-
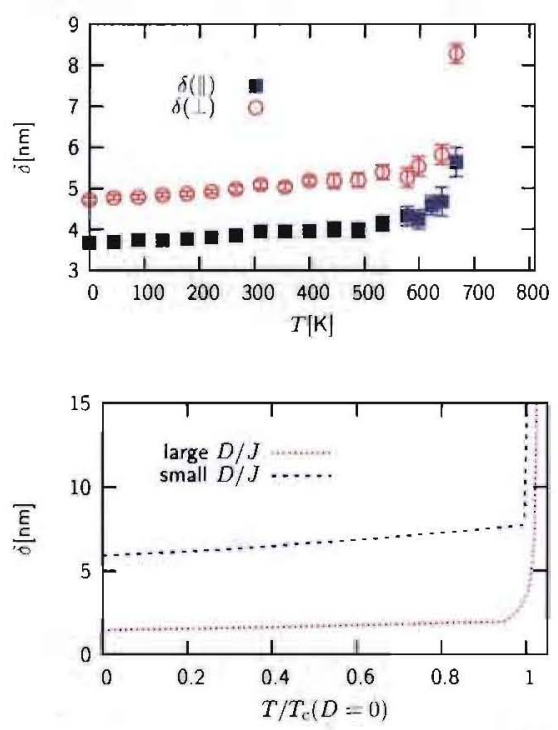

FIG. 6. (Color online) Domain wall width $\delta$ versus temperature. The upper figure shows results for the atomistic FePt model for two different domain wall orientations. The lower figure shows results for the MF approximation for two different values of the anisotropy. 
nite temperature, their values differ, which indicates an elliptical wall profile. At room temperature, the transverse component of the magnetization is about $5 \%$ smaller than the easy-axis component. At a certain temperature close to but below the Curie temperature, $h_{t}$ vanishes while $h_{z}$ remains finite. Obviously, the two amplitudes define two distinct order parameters which vanish continuously at two different temperatures: the upper one being the usual Curie temperature $T_{C}$ and the lower one a second critical temperature $T_{h}$, which describes the phase transition of the transverse component of the magnetization. For temperatures $T_{t}<T<T_{C}$, the domain wall is linear with an easy-axis component of the magnetization only. The data from MF calculation show this behavior even more clearly and without any finite-size effects which-as usual-lead to a rounding of the transition.

The corresponding domain wall widths are shown in Fig. 6. In the FePt model, the domain wall width depends clearly on the orientation of the domain wall. As discussed in Ref. 7, the orientation dependence is due to the fact that the $\mathrm{Fe}-\mathrm{Fe}$ exchange interaction is stronger within the Fe planes than perpendicular to the planes where the interaction is via the $\mathrm{Pt}$ sites. An additional temperature dependence of the domain wall width can be found both in our atomistic FePt simulations and in the MF approximation. First, the width increases slightly with temperature. At the first critical temperature $T_{k}$, the wall width is nonanalytic, exhibiting a discontinuous change in gradient, this being most pronounced for large $D / J$. The reason for that is discussed in Refs. 1, 2, and 5, where the anisotropy dependence of the two critical temperatures is investigated in more detail. One finding in these papers is that the difference between both critical temperatures $T_{h}$ and $T_{C}$ scales with the squared inverse domain wall width $\delta,\left(T_{C}-T_{h}\right) /\left(T_{C}\right) \sim \delta^{-2}$.

The wall width finally diverges at the Curie temperature. Once again, the data from MF approximation show this behavior even more clearly than the atomistic simulations and provide strong support for our interpretation of the behavior of the atomistic model.

\section{B. Domain wall energies}

In the following, we investigate to what degree our findings affect the energy of the domain walls. Therefore, we calculate the domain wall energies from energy differences between systems with and without domain wall, i.e., either simulated with antiparallel or with parallel fixed boundary conditions. We calculate both the internal domain wall energy $\Delta E / J$ and the free domain wall energy $\Delta F / J$.

The free energy of the domain walls is calculated using the equation

$$
\Delta F(\beta)=\frac{1}{\beta} \int_{0}^{\beta} \Delta E\left(\beta^{\prime}\right) d \beta^{\prime},
$$

with $\beta=1 / k_{B} T$. Since above $T_{C}$ the energies of systems with and without a wall are identical, it is sufficient to start the integration at or close to the Curie temperature. The internal energy is calculated as time average of the Hamiltonian [Eq. (1)].

In the upper part of Fig. 7, these energy differences are
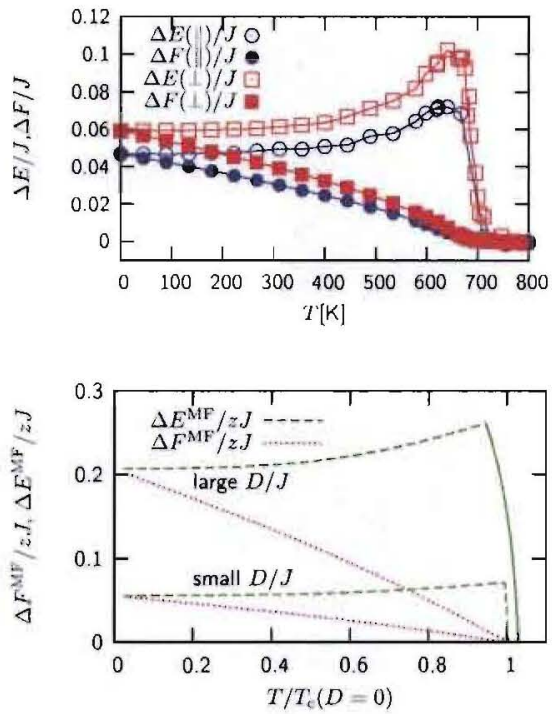

FIG. 7. (Color online) Domain wall energy and free energy versus temperature for the two different orientations of the domain wall (above). The figure below shows results from MF calculations. The curves for $D=0.01442 J$ are comparable with $\Delta F$ and $\Delta E$ from the FePt simulation.

shown versus temperature for both domain wall orientations. A remarkable finding is that the internal energy $\Delta E / J$ increases with increasing temperature until it reaches a maximum before it finally decreases rapidly and vanishes at the Curie temperature. It is reasonable to assume that this peak occurs at the lower critical temperature $T_{h}$ marking the transition from elliptical to linear walls.

In order to verify this assumption, we also calculate the domain wall energies with the aid of the MF approximation. After having solved the self-consistency equations, the internal energy is calculated directly from the MF Hamiltonian [Eq. (3)], and the free energy is calculated as $F=-k_{B} T \ln Z$. These results are shown in the lower part of Fig. 7, once again for two different values of the anisotropy. As mentioned before, the data for the lower anisotropy, $D / J$ $=0.01442$, are compatible with our values for the FePt LLG calculations. The MF approximation results show clearly a peak in the intemal domain wall energy. This peak appears exactly at that temperature $T_{h}$ where we have found the first appearance of a linear wall before (for comparison, see Fig. 4). The agreement between atomistic and MF approximation results confirms our assumption that the peak indicates the transition from an elliptical to a linear domain wall. The figure also demonstrates that the transition from elliptical to linear walls depends on the strength of the anisotropy $D / J$, or, more exactly on the domain wall widths. This dependence is discussed in detail in Ref. 5.

Using our data for the DW free energy and domain wall width, calculated as explained before, we are able to calculate the thermodynamic exchange stiffness $A(T)$ and the uniaxial anisotropy constant $K(T)$, suitable as FePt material parameters for a finite temperature micromagnetic continuum theory. However, due to the orientation dependence of the DW properties in this layered ferromagnet, different 


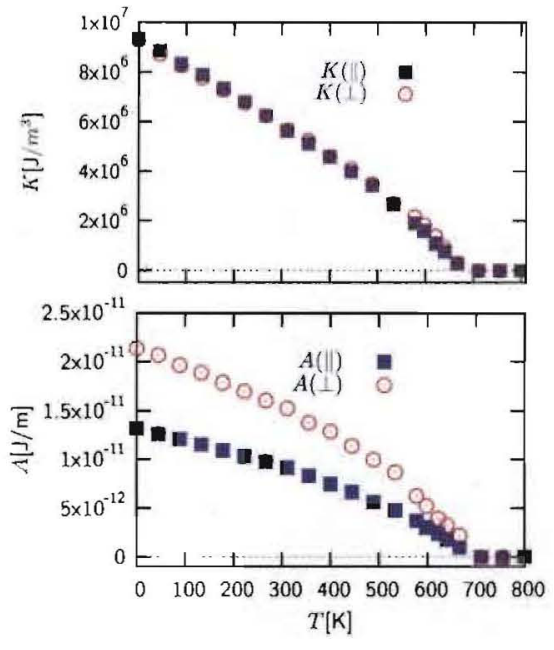

FIG. 8. (Color online) Temperature dependence of the uniaxial anisotropy constant and the exchange stiffness for FePt.

exchange stiffnesses will appear for different directions. Using the equations $\delta_{l}=\pi \sqrt{A_{l} / K_{l}}$ and $\Delta F_{l}=4 \sqrt{A_{l} K_{l}}$, with $l=\perp$, $\|$, we can calculate the (anisotropic!) micromagnetic exchange stiffness $A_{\perp}$ for the $x$ and $y$ components of a Cartesian coordinate system and $A_{\|}$for the $z$ (easy-axis) component. The numerical values we obtained are shown in Fig. 8. Note that $A_{\perp}$ and $A_{\|}$differ by a factor of 1.6, while the values of the uniaxial anisotropy parameter depend at most weakly on the wall orientation. Both quantities vanish at the critical temperature. Also, the values of the temperature dependent uniaxial anisotropy parameter coincide (apart from finite-size effects) with values obtained earlier with different methods, ${ }^{12}$ underpinning the validity of our approach.

\section{CONCLUSIONS}

We considered the temperature dependence of domain wall properties in FePt. Due to the high anisotropy of FePt and its small domain wall width, it is possible to detect three different kinds of domain wall profiles occurring at different temperatures. At zero temperature, we find circular Bloch walls which become elliptical for any finite temperature. We were able to detect the transition from elliptical to linear walls with the aid of the domain wall profiles and the internal energies. In this context, the unusual behavior of the internal energy having a nonanalytical behavior below the Curie temperature is of special importance. The transition to linear wall sets in approximately $50 \mathrm{~K}$ below the Curie temperature. Our results suggest that due to its small domain wall widths, FePt is a possible candidate for the experimental observation of elliptical or even linear domain wall structures.

Micromagnetic theory as formalized by Brown ${ }^{18}$ is founded on thermodynamic principles, recognizing that the physics of the problem requires minimization of the free energy rather than the internal energy. Brown, in fact, explicitly discussed two approaches to the calculation of free energies. The first is a phenomenological method involving a truncated series expansion, use of symmetry considerations, and experimental evaluation of the relevant parameters. The second he referred to as "the microscopic method," essentially a detailed model capable of predicting desired properties by statistical mechanics. Although Brown commented that the latter approach is "not usually attempted because the mathematical difficulties are too great," modern techniques and computational resources are sufficient, as shown here, to tackle the problem. Because of the complexity of determining the free energy, present day numerical micromagnetics generally proceed via the minimization of the internal energy rather than the free energy. This approach has continued into present day numerical micromagnetics. A further assumption of micromagnetics is the constraint of constant $\|\mathbf{M}\|$. Here, we demonstrate that this constraint is not necessarily fulfilled in domain walls at nonzero temperatures. Not only are there local thermal fluctuations in $\|\mathbf{M}\|$, but in elliptical and linear walls, even the average $\|\mathbf{M}\|$ becomes a function of position. By comparing our thermodynamic results with micromagnetic solutions, we were able to compute the parameters $A_{\mid}$, $A_{\perp}$, and $K$ that appear in the free energy. Although the micromagnetic solutions [Eqs. (5) and (6)] used in the comparison assume that $\|\mathbf{M}\|$ is independent of position, the agreement between the two $K$ solutions in Fig. 8 suggests that the comparison is valid over most of the temperature range. The small residuals at high temperature are probably due to the loss of constancy of $\|\mathbf{M}\|$. At high temperatures, a general micromagnetic approach should allow the removal of the constraint of constant $\|\mathbf{M}\|$. We have previously shown ${ }^{9}$ that the Landau-Lifshitz-Bloch (LLB) equation encapsulates to a remarkable degree the underlying physics as described by the atomistic model. Thus, we suggest the use of the LLB equation rather than the LLG equation as the natural extension of micromagnetics for high temperatures. $11,19.20$

\section{ACKNOWLEDGMENTS}

D.H. and N.K. acknowledge financial support by Seagate Research, Pittsburgh, PA. O.N.M. is grateful to SFI during his stay at Trinity College, Dublin. The use of the White Rose Grid computational resources at the Universities of York and Sheffield is gratefully acknowledged. 
*dsh503@york.ac.uk

${ }^{1}$ L. N. Bulaevskir and V. L. Ginzburg, Sov. Phys. JETP 18, 530 (1964).

${ }^{2}$ D. A. Garanin, Physica A 172, 470 (1991).

${ }^{3}$ Y. Labaye, L. Berger, and J. M. D. Coey, J. Appl. Phys. 91, 5341 (2002)

${ }^{4}$ J. M. D. Coey, L. Berger, and Y. Labaye, Phys. Rev. B 64 020407 (R) (2001).

${ }^{5}$ N. Kazantseva, R. Wieser, and U. Nowak, Phys. Rev. Lett. 94, 037206 (2005).

${ }^{6}$ R. Wieser, K. D. Usadel, and U. Nowak, Phys, Rev. B 74, 094410 (2006).

${ }^{7}$ D. Hinzke, U. Nowak, O. N. Mryasov, and R. W. Chantrell, Appl. Phys. Lett. 90, 082507 (2007).

${ }^{8}$ J. Kötzler, D. A. Garanin, M. Hartl, and L. Jahn, Phys. Rev. Lett. 71, 177 (1993).

${ }^{9}$ M. Laufenberg, D. Backes, W. Bührer, D. Bedau, M. Kläui, U. Rüdiger, C. A. F. Vaz, J. A. C. Bland, L. J. Heyderman, F. Nolting, S. Cherif, A. Locatelli, R. Belkhou, S. Heun, and E. Bauer, Appl. Phys. Lett. 88, 052507 (2006).
${ }^{10}$ M. Hartl-Malang, J. Kötzler, and D. A. Garanin, Phys. Rev. B 51, 8974 (1995).

"O. Chubykalo-Fesenko, U. Nowak, R. W. Chantrell, and D. Garanin, Phys. Rev. B 74, 094436 (2006)

${ }^{12}$ O. N. Mryasov, U. Nowak, K. Guslienko, and R. W. Chantrell, Europhys. Lett. 69, 805 (2005).

${ }^{13}$ O. N. Mryasov, V. A. Gubanov, and A. I. Liechtenstein, Phys. Rev, B 45, 12330 (1992).

${ }^{14}$ A. B. Shick and O. N. Mryasov, Phys. Rev. B 67, 172407 (2003)

${ }^{15}$ O. N. Mryasov, J. Magn. Magn. Mater. 272-276, 800 (2004).

${ }^{16}$ U. Nowak, O. N. Mryasov, R. Wieser, K. Guslienko, and R. W. Chantrell, Phys. Rev, B 72, 172410 (2005).

${ }^{17}$ U. Nowak, in Annual Reviews of Computational Physics IX, edited by D. Stauffer (World Scientific, Singapore, 2001), p. 105.

${ }^{18}$ W. F. Brown, Micromagnetics (Wiley, New York, 1963).

${ }^{19}$ D. A. Garanin, Phys. Rev. B 55, 3050 (1997).

${ }^{20}$ U. Atxitia, O. Chubykalo-Fesenko, N. Kazantseva, D. Hinzke, U. Nowak, and R. W. Chantrell, Appl. Phys. Lett. 91, 232507 (2007). 